\title{
Effects of milk powder intervention on bone mineral density and indicators related to bone metabolism in Chinese adolescents
}

\author{
J.X. Lu ${ }^{1} \cdot$ H. Pan ${ }^{1} \cdot$ X.Q. Hu ${ }^{1} \cdot$ Z.W. Huang ${ }^{1} \cdot$ Q. Zhang ${ }^{1}$
}

Received: 13 December 2018 / Accepted: 21 July 2019 / Published online: 2 August 2019

(C) The Author(s) 2019

\begin{abstract}
Summary The study is to determine the effects of milk powder on bone density and metabolism in healthy adolescents. Vitamin $\mathrm{D}$ and calcium supplements increased IGF-1 but did not affect bone mineralization or turnover. Higher vitamin D in combination with sufficient calcium supplementation in such populations requires attention.

Introduction Both calcium and vitamin D play an important role in bone mineralization in adolescents.

Methods In this one and a half-year randomized controlled trial, 232 participants (aged 12-15 years) were randomly assigned to three intervention groups receiving milk powder fortified with vitamin D $400 \mathrm{IU}$ plus calcium 300 , or 600 , or $900 \mathrm{mg}$ [Ca3D $(n=$ 54), $\mathrm{Ca} 6 \mathrm{D}(n=56)$, and Ca9D $(n=49)$ ], or one control group maintaining habitual diet [control $(n=73)]$. Bone turnover markers, serum intact PTH, 25(OH)D, and IGF-1 levels were measured at baseline and one and a half years, and bone mineral contents and bone areal mineral density were measured by Dual-energy X-ray absorptiometry (DXA) at baseline, one year, and one and a half years.

Results Baseline average serum 25(OH)D level and calcium intake were $29.4 \mathrm{nmol} / \mathrm{L}$ and $363.5 \mathrm{mg} /$ day, respectively. There was a significant increase in bone turnover, total body, hip, lumbar spine bone mineral density (BMD), and total body BMC as well as slight fluctuations in 25(OH)D levels over one and a half years without between-group differences. Significantly decreased serum PTH level was only observed in the Ca6D group $(31 \%, p<0.0001)$, in which the intervention effect was also significant $(p=$ $0.0029)$ compared with the control group. IGF-1 levels increased significantly in all intervention groups (18.5 to $22.8 \%, p<0.05)$ but decreased in the control group $(16.5 \%, p<0.05)$, and the group by time interaction was also significant $(p=0.0029)$.

Conclusions Our study showed that in healthy adolescents with low vitamin D status and calcium intake, mild vitamin D and mild to modest calcium supplements increased IGF-1 but did not affect bone mineralization or turnover. Higher vitamin D in combination with sufficient calcium supplementation in such populations requires attention.
\end{abstract}

Keywords 25-hydroxyvitamin D · Adolescents $\cdot$ Bone density $\cdot$ Calcium $\cdot$ Insulin-like growth factor- 1 Parathyroid hormone

\section{Introduction}

Insufficient calcium intake has been shown to have an adverse impact on bone density in healthy adolescents [1,2], and this negative effect can be resolved by calcium supplementation [3]. However, the positive supplementation effects were questioned in a meta-analysis study [4], which reported a small (2\%) effect on the BMD of the total body but not lumbar

Q. Zhang

zhangqian7208@163.com

1 National Institute of Nutrition and Health, Chinese Center for Disease Control and Prevention, 29 Nan Wei Road, Xi Cheng District, Beijing 100050, China spine and hip, the sites related to the most important fractures in later life. Given the fact that studies included in the metaanalysis have some limitations such as older technology for measuring BMD and no bone turnover markers used, a study was conducted to evaluate the effect of calcium supplementation on bone mineral accretion excluding these limitations, which found a beneficial effect on total body, lumbar spine, and total hip BMD [5]. To our knowledge, studies in the Chinese population are limited.

Vitamin D is also an essential dietary factor in the maintenance of normal mineralization of skeletal tissue [6]. Even though the relation between vitamin D deficiency and rickets has been well revealed, the possible association between vitamin $\mathrm{D}$ and bone health remains controversial in adolescents. A prospective study indicated that the maximization of peak 
bone mass might be affected adversely by lower vitamin $\mathrm{D}$ in pubertal girls [7]. In addition, one study suggested that low $25(\mathrm{OH}) \mathrm{D}$ status could aggravate the negative effect of low calcium intake on bone mineralization in adolescent girls [8]. Furthermore, a randomized controlled trial has shown that participants receiving milk fortified with calcium and cholecalciferol had a greater increase in percentage change in total body BMD than those receiving milk fortified with only calcium in adolescents with lower vitamin D status at baseline [9]. A meta-analysis further indicated that vitamin D supplements were unlikely to have a beneficial effect in adolescents with normal vitamin D levels but only in those with deficient vitamin D levels [10]. Thus it seems important to maintain both sufficient calcium intake and vitamin D status to ensure optimal skeletal health in adolescents with low vitamin D level.

Bone turnover markers can reflect either bone resorption or formation. TRAP- $5 \mathrm{~b}$ can respond to bone resorption, ALP can respond to bone formation, $\mathrm{PTH}$ and $25(\mathrm{OH}) \mathrm{D}$ regulate the whole bone metabolism process, and IGF-1 promotes bone synthesis. The growth of adolescent bones is done by their joint action.

In this randomized controlled study, we performed a supplementation of milk powder fortified with calcium and vitamin D in Chinese adolescents for one and a half years. Serum bone turnover markers, IGF-1, PTH status (at baseline and one and a half years later), and BMD of total body, lumbar spine, and total hip (at baseline, one year, and one and a half years) were measured. We examined the prevalence of vitamin $\mathrm{D}$ deficiency, and prospective analyses of these repeated measurement data were used to assess the effects of calcium and vitamin D-fortified milk powder on bone density and biomarkers in Chinese adolescents living in the rural area of Beijing, China.

\section{Participants and methods}

\section{Participants and recruitment}

Cluster sampling was used to attain the study sample in this randomized controlled trial. Three secondary schools were randomly selected in one rural district in Beijing, China, and then four classes in first grade were randomly selected in each school as participants. To be eligible for the trial, participants had to be $12-15$ years of age and to be free of the following diseases in the one year prior to enrollment: musculoskeletal diseases, liver or kidney disorders, or other endocrinosis, such as diabetes and hyperthyroidism. In addition, participants were excluded from the trial if they were taking vitamin D or calcium supplements. Of the 286 participants recruited, 31 were not eligible, and 23 participants withdrew before the trial officially started. Therefore, 232 healthy Chinese adolescents
(115 boys and 117 girls; 11.9 to 15.2 years of age) were recruited for an interventional trial of milk powder fortified with vitamin D and calcium from December 2009 to June 2011 (early fall and early spring in Beijing).

\section{Randomization of vitamin D and calcium supplements}

Twelve first-grade classes from three secondary schools in rural Beijing were randomized to one control group (consuming habitual diets) and three study groups (vitamin D and calcium supplements), matched for BMD at baseline by class. The details are as follow: One class in each school was randomized to the control group at first. After that, in the remaining nine classes, the participants were randomized to three study groups in a double-blind fashion, namely, $400 \mathrm{IU} /$ day vitamin D plus a different dosage of calcium per day of 300 (Ca3D group), 600 (Ca6D group), and 900 (Ca9D group) mg. The supplement was performed in the form of packed milk powder (provided by Nestle, China) labeled with capitals A, $\mathrm{B}$, and $\mathrm{C}$, and only the manufacturers knew the difference of the calcium content. One pack of milk powder $(20 \mathrm{~g})$ containing $200 \mathrm{IU}$ vitamin $\mathrm{D}$ and 150,300 , or $450 \mathrm{mg} \mathrm{CaCO}_{3}$ was prepared by parents for participants of three different study groups in the morning and evening each day and participants had to drink them under parents' intendance. The nutritional composition of the daily supplemented test milk powder $(40 \mathrm{~g})$ is shown in Table 1 . The study milk powder was dispensed once a month over one and a half years, and the storage and blinded distribution of it were conducted by the Institute for Nutrition and Food Safety, Chinese Center for Disease Control and Prevention (now renamed the National Institute of Nutrition and Health, Chinese Center for Disease Control and Prevention).

\section{Dietary intake}

At baseline, An experienced nutritionist and several trained investigators assessed usual dietary intakes using consecutive

Table 1 Nutritional composition of the test milk powder (per $40 \mathrm{~g}$ )

\begin{tabular}{lll}
\hline & & RNI \\
\hline Energy (kJ) & 163 & $9079 / 8088$ (male/female) \\
Protein (g) & 10.2 & 75 \\
Fat (g) & 4.7 & $53.7 \sim 64.4^{\Delta}$ \\
Carbohydrate (g) & 20.0 & $271.1 \sim 302.5^{\triangle}$ \\
Dietary fiber (g) & 3.6 & $25.9^{\triangle}$ \\
Vitamin D (IU) & 400 & 200 \\
Calcium (mg) & $300 / 600 / 900$ & $1000^{*}$ \\
\hline
\end{tabular}

RNI, recommended nutrient intakes

${ }^{\Delta}$ RNI for people with energy intake of $8088 \mathrm{~kJ}$

*Adequate intakes (AI) for calcium intake 
three-day food records (including two weekdays and one weekend day), including meals and snack. The quantification of food items was performed by Chinese measures of bowls, plates, and spoons of standard size with the assistance of a set of food measure models. Nutrient intakes were calculated using the Chinese Food Composition Tables published in 2002 [11] and 2004 [12].

\section{Anthropometry and bone mineral density}

Height was measured to the nearest $0.1 \mathrm{~cm}$ using a stadiometer (Jianmin, Beijing, China). Weight was measured to the nearest $0.1 \mathrm{~kg}$ using electronic weight scales (Thinner; Measurement Specialities, Fairfield, NJ, USA), with participants wearing light clothing and no shoes. Body mass index (BMI) was calculated by the equation BMI $\left(\mathrm{kg} / \mathrm{m}^{2}\right)=$ weight $(\mathrm{kg}) /$ height $\left(\mathrm{m}^{2}\right)$. Total body BMD and BMC were measured using the dual-energy X-ray absorptiometry (DXA) via a Norland XR46 densitometer (Norland Medical Systems, Fort Atkinson, WI, USA). Measurements were made at the Beijing Sport University, Beijing, at three time points (initial baseline, one year, and one and a half years) by two experienced technicians throughout the trial. All bone data was analyzed by the same technician using the Norland enhanced software version 3.9.4. A daily quality assurance test was performed with a manufacturer-supplied phantom, and the accuracy error of repeated measurements was $<1.0 \%$. Prior to the first scan of each time, the densitometer was calibrated in accordance with the manufacturer's recommendations.

\section{Biochemical analyses}

One 5-ml overnight fasting venous blood sample was collected between 6.30 and 9.00 a.m. during the period March to April at the end of fall. The plasma was separated and stored at $-80{ }^{\circ} \mathrm{C}$ prior to analysis. Plasma $25(\mathrm{OH}) \mathrm{D}$ concentration was determined by ${ }^{125}$ I-radioimmunoassay (RIA) kits (Diasorin, Antony, France), which involved only a preliminary extraction without a chromatographic purification step, followed by the addition of antibody and tracer specific for the $25(\mathrm{OH}) \mathrm{D} 3$ metabolite. The limit of detection of the assay is $5 \mathrm{nmol} / \mathrm{L}$ and the cross-reactivity for $25(\mathrm{OH}) \mathrm{D}$ is $100 \%$. The intra- and inter-assay CVs were $4.2 \%$ and $6.6 \%$ for $25(\mathrm{OH}) \mathrm{D}$. The serum bone-specific alkaline phosphatase (ALP), insulinlike growth factor-1 (IGF-1), intact parathyroid hormone (PTH), and tartrate-resistant acid-alkaline phosphatase 5b (TRAP-5b) were measured using a commercial ELISA kit method (IDS Ltd., Boldon, Tyne and Wear, UK). The intraand inter-assay CVs were 10\%, 8.9-12.9\%, 10\%, and 10\%, respectively. All samples were assayed in duplicate using the same batch of kits.

\section{Statistical analysis}

Descriptive results are reported as mean $\pm \mathrm{SD}$ and differences as mean \pm SE unless otherwise indicated. Study participants characteristics at baseline were tested using the Ridit (relative to an identified distribution unit) test for categorical variables, one-way analysis of variance (ANOVA) for continuous variables, and the Wilcoxon rank sum test for non-normally distributed continuous variables. A mixed linear model was used to determine treatment, time and interaction effects on these bone-related outcomes adjusting for baseline values, pubertal stages, and sex, with time since baseline as the timeline, group effects as fixed, and participant effects as random and a first-order autoregressive covariance structure (AR1). If the linear mixed model analysis showed a significant group by time interaction, pairwise comparisons for the group by time interaction were evaluated by the model. If the linear mixed model analysis showed a significant time effect, the time effects at one and one and a half years were further evaluated by the model. A two-sided $p$ value $\leq 0.05$ was considered statistically significant. Analyses were performed with SAS, Version 9.0 (SAS Institute Inc., Cary, NC, USA).

\section{Results}

\section{Participant characteristics}

Figure 1 gives the flow through the study for participants. The characteristics of 232 study participants aged 11.9 to 15.2 years at baseline are shown in Table 2. There were no significant differences between groups in baseline characteristics including average age, height, BMI, dietary calcium intake, and proportion of pubertal stage. Average daily calcium intakes were insufficient for participants in all groups with a range of 32 to $41 \%$ of the Recommended Dietary Intake (AI, $1000 \mathrm{mg} /$ day) for adolescents aged 11 to 18 years [13]. Total calcium intakes (dietary plus supplement calcium) were significantly different between groups, and the sequence was in accordance with the quantity of calcium supplements $(\mathrm{Ca} 9 \mathrm{D}>\mathrm{Ca} 6 \mathrm{D}>\mathrm{Ca} 3 \mathrm{D}>$ control). Vitamin D supplements were significantly higher in three intervention groups than in the control group while no difference between interventional groups (Table 2). There were $55 \%$ or $7 \%$ of participants having serum $25(\mathrm{OH}) \mathrm{D}$ level ranged 20 up to $30 \mathrm{ng} / \mathrm{mL}$ or less than $20 \mathrm{ng} / \mathrm{mL}$, indicating either insufficient or deficient vitamin D status [14]. During the one and a half years, 11 $(15.0 \%), 6(12.2 \%), 2(3.6 \%)$, and $6(11.1 \%)$ participants withdrew from the trial in control, Ca3D, Ca6D, and Ca9D, respectively. No significant difference between 


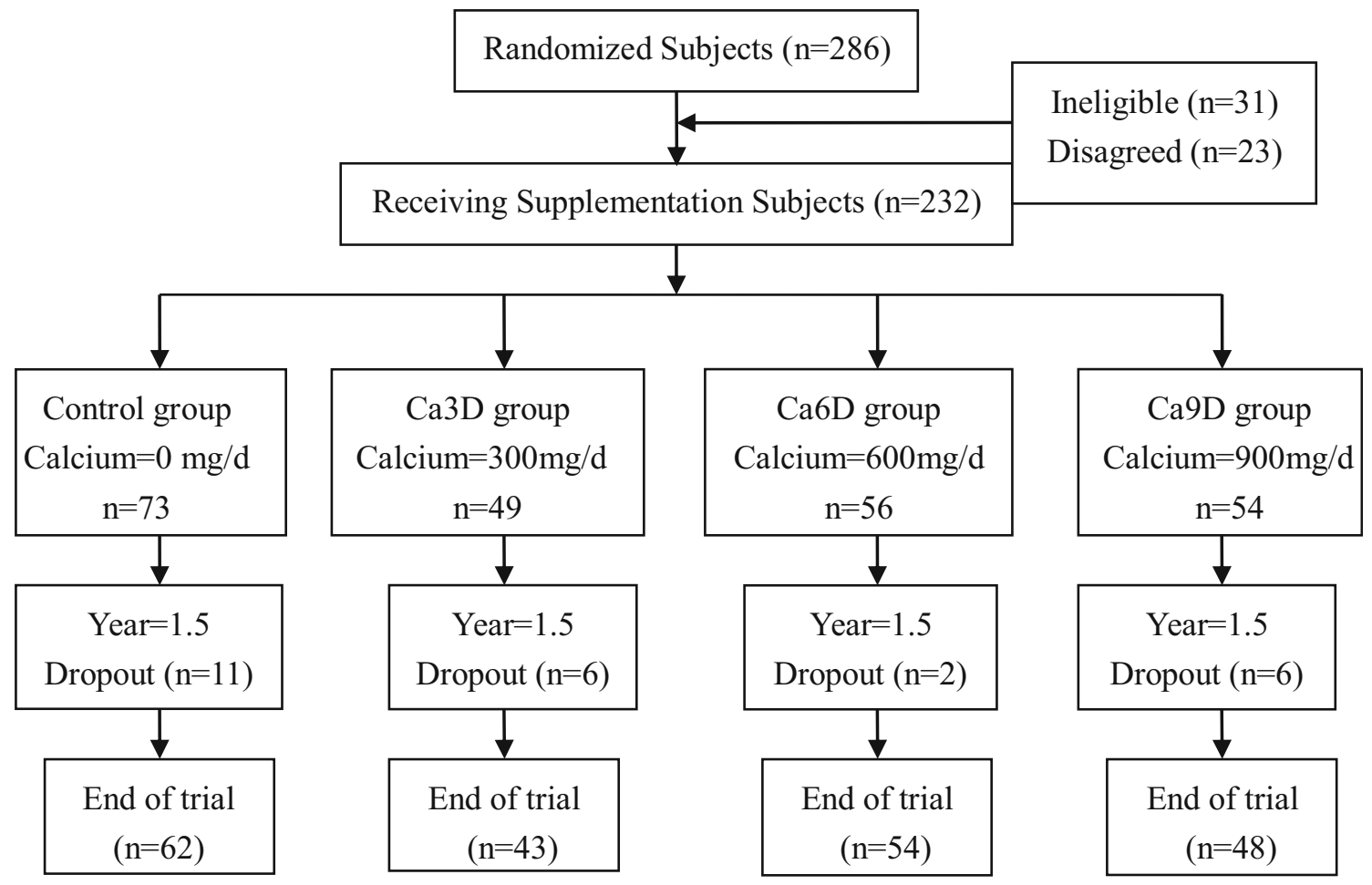

Fig. 1 Flow diagram of the progress through phases of the trial

groups was observed in the number of participants lost to follow up. There was no significant difference in the compliance rate with the test milk powder between intervention groups $(81.3 \%, 77.9 \%$, and $73.7 \%$ for $\mathrm{Ca} 3 \mathrm{D}, \mathrm{Ca} 6 \mathrm{D}$, and Ca9D, respectively).

\section{Effects of intervention on 25(OH)D, ALP, and TRAP-5b}

Serum 25(OH)D and ALP levels in all groups at baseline were not significantly different, while the TRAP- $5 \mathrm{~b}$ status in the $\mathrm{Ca} 6 \mathrm{D}$ group was higher than those in control and Ca9D

Table 2 Characteristics of the participants at baseline

\begin{tabular}{|c|c|c|c|c|c|}
\hline & Control & Ca3D & Ca6D & Ca9D & $p$ value \\
\hline$N$ (female) & $73(37)$ & $49(26)$ & $56(24)$ & $54(30)$ & 0.5745 \\
\hline Age (years) & $13.0 \pm 0.7$ & $13.0 \pm 0.7$ & $13.1 \pm 0.6$ & $13.2 \pm 0.6$ & 0.5118 \\
\hline Height (cm) & $155.6 \pm 6.9$ & $156.4 .1 \pm 6.7$ & $155.5 \pm 8.0$ & $154.2 \pm 8.5$ & 0.4752 \\
\hline BMI $\left(\mathrm{kg} / \mathrm{m}^{2}\right)$ & $19.2 \pm 3.4$ & $19.9 \pm 4.2$ & $18.6 \pm 3.1$ & $19.2 \pm 3.5$ & 0.3731 \\
\hline Pubertal stage, $N(\%)$ & & & & & 0.8264 \\
\hline I & $4(5.63)$ & $5(9.80)$ & $5(8.77)$ & $5(8.47)$ & \\
\hline II & $27(38.03)$ & $13(25.49)$ & $23(40.35)$ & $16(27.12)$ & \\
\hline III & $26(36.62)$ & $22(43.14)$ & $20(35.09)$ & $33(55.93)$ & \\
\hline IV & $14(19.72)$ & $10(19.61)$ & $9(15.79)$ & $4(6.78)$ & \\
\hline $\mathrm{V}$ & $0(0)$ & $1(1.96)$ & $0(0)$ & $1(1.69)$ & \\
\hline Calcium intake (mg) & $315.8 \pm 260.9$ & $372.6 \pm 265.9$ & $372.5 \pm 239.9$ & $410.2 \pm 318.2$ & 0.2481 \\
\hline \multicolumn{6}{|l|}{ Biochemistry } \\
\hline $\operatorname{ALP}(\mathrm{U} / \mathrm{L})$ & $60.02 \pm 30.58$ & $58.44 \pm 21.91$ & $61.16 \pm 25.15$ & $54.98 \pm 22.69$ & 0.6120 \\
\hline TRAP-5b (U/L) & $4.18 \pm 2.67^{\mathrm{a}}$ & $4.76 \pm 3.16^{\mathrm{a}, \mathrm{b}}$ & $5.69 \pm 3.54^{\mathrm{b}}$ & $4.11 \pm 2.29^{\mathrm{a}}$ & 0.0110 \\
\hline IGF-1 (ng/mL) & $789.14 \pm 372.40$ & $751.65 \pm 428.89$ & $693.56 \pm 423.73$ & $666.57 \pm 327.42$ & 0.2708 \\
\hline $25(\mathrm{OH}) \mathrm{D}(\mathrm{nmol} / \mathrm{L})$ & $29.51 \pm 9.10$ & $29.93 \pm 7.94$ & $28.58 \pm 8.89$ & $29.66 \pm 8.95$ & 0.8615 \\
\hline PTH (pg/mL) & $77.26 \pm 44.50$ & $89.07 \pm 39.59$ & $99.16 \pm 63.31$ & $84.71 \pm 45.12$ & 0.0819 \\
\hline
\end{tabular}

a, b Horizontal comparison of differences between groups; the same letter has no difference, different letters have differences 
Table 3 The mixed linear model for changes in serum bone turnover markers, PTH, IGF-1, and $25(\mathrm{OH}) \mathrm{D}$ levels over one and a half years (mean $\pm \mathrm{SE}$ )

\begin{tabular}{lllllll}
\hline & Control & Ca3D & Ca6D & Ca9D & \multicolumn{2}{l}{$p$ value } \\
\cline { 5 - 6 } & $(N=62)$ & $(N=43)$ & $(N=54)$ & $(N=48)$ & Time & $\begin{array}{c}\text { Group by } \\
\text { time }\end{array}$ \\
\hline $\begin{array}{c}25(\mathrm{OH}) \mathrm{D} \\
(\mathrm{nmol} / \mathrm{L})\end{array}$ & $-2.75 \pm 1.14 *$ & $-0.73 \pm 1.46$ & $-0.30 \pm 1.19$ & $0.29 \pm 1.39$ & 0.2553 & 0.2588 \\
$\begin{array}{c}\mathrm{ALP}(\mathrm{U} / \mathrm{L}) \\
\begin{array}{c}\text { TRAP-5b } \\
(\mathrm{U} / \mathrm{L})\end{array}\end{array}$ & $8.93 \pm 5.77$ & $12.08 \pm 5.87$ & $21.46 \pm 6.88^{*}$ & $8.99 \pm 5.89$ & $<0.0001$ & 0.3697 \\
\hline
\end{tabular}

25(OH)D: 0.2521, 0.1010, 0.0802 pairwise comparisons

Adjusted for baseline value, sex, and baseline pubertal stage

* Significantly different from that of baseline, $p<0.05$ groups but not in the Ca3D group (Table 2). Changes in serum 25(OH)D, ALP, and TRAP-5b levels throughout interventional duration were presented in Table 3. Linear mixed-effects model did not indicate significant group by time interactions for all of them while significant time effect for ALP and TRAP-5b ( $p<0.0001$ for both of them) but not for $25(\mathrm{OH}) \mathrm{D}$. Serum 25(OH)D levels had a decrease in control, $\mathrm{Ca} 3 \mathrm{D}$, and Ca6D groups while a slight increase in the Ca9D group; only the change, however, in the control group was statistically significant $(p<0.05)$. ALP levels were higher than those at baseline in all groups while significance was only found in the Ca6D group, and between-group differences were not significant. Similarly, TRAP-5b levels were significantly increased across all groups $(p<0.05)$ whereas no difference was found between groups.

\section{Effects of intervention on PTH and IGF-1}

There was no significant difference between groups for both PTH and IGF-1 levels in baseline values (Table 2). The mixed linear model indicated a significant group by time interaction and time effect for both PTH and IGF-1 (Fig. 2a, b). PTH levels were decreased in all intervention groups but increased in the control group; however, only the decrease in the Ca6D group was significant $(31.2 \%, p<0.0001)$. Pairwise comparisons showed significant treatment effects for the Ca6D group compared with other groups $(p=0.0006,0.0286$, and 0.0064 in comparison with control, $\mathrm{Ca} 3 \mathrm{D}$, and $\mathrm{Ca} 9 \mathrm{D}$ groups, respectively). IGF-1 levels were significantly lower than that at baseline in the control group $(16.2 \%, p<0.05)$ while higher in all intervention groups $(18.5 \%, 22.6 \%$, and $22.8 \%$ for Ca3D, $\mathrm{Ca} 6 \mathrm{D}$, and $\mathrm{Ca} 9 \mathrm{D}$ groups, respectively, $p<0.05$ for all of them), in which IGF-1 levels increased but decreased in the control group. In contrast, further pairwise comparisons indicated significant treatment by time interaction for all intervention groups compared with the control group $(p=0.0046$, 0.0029, and 0.0022 for Ca3D, Ca6D, and Ca9D groups compared with the control group, respectively).

\section{Effects of intervention on bone}

No significant differences were found between groups in $\mathrm{BMD}$ and BMC at all measured sites at baseline (Table 4). Total body, lumbar spine, total hip areal BMD, and total body BMC increased significantly from baseline at one and one and a half years in all groups (Table 4). There was no significant group by time interaction for any of bone variables when the linear mixed model was performed regardless of adjustment for baseline value, sex, and baseline pubertal stage or not, suggesting that there was no treatment effect, which was also not influenced by those variables adjusted.

\section{Discussion}

Bone resorption and formation are mutually restricted to achieve dynamic balance to promote bone growth in an adolescent. PTH and 25(OH)D play a regulatory role, and IGF-1 promotes bone resorption and formation. Our study suggested that, in adolescent boys and girls with poor serum $25(\mathrm{OH}) \mathrm{D}$ level, there was no significant difference in changes in the total body BMC and BMD at total body, lumbar spine, and total hip over one and a half years in the group who received habitual diet compared with groups who received milk powder fortified with $400 \mathrm{IU}$ vitamin D and 300, 600, or $900 \mathrm{mg}$ calcium. The intervention increased the IGF-1 level for all groups while only decreased the PTH level and increased bone formation in the group who received milk powder fortified with 400 IU vitamin D and $600 \mathrm{mg}$ calcium but did not affect bone resorption.

\section{Effects on serum 25(OH)D}

In the present study, the control group had a $9.3 \%$ decrease in the $25(\mathrm{OH}) \mathrm{D}$ level after one and a half years compared with intervention groups, ranging from -2.4 to $1.0 \%$. These 

$\square$ Control group
$\mathbb{N}$ Ca3D group
血Ca6D group
Ca9D group

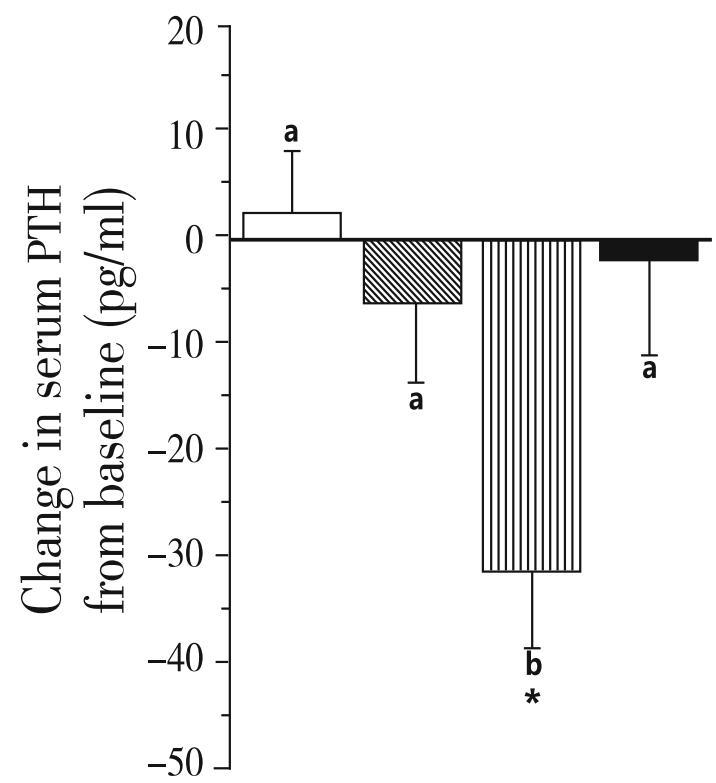

Fig. 2 a Change in serum intact PTH from baseline (error bars denote standard error). There was a significant group by time interaction and time effect, $p=0.0045$ and 0.0068 , respectively. $N=62,42,53$, and 48 for control, Ca3D, Ca6D, Ca9D groups, respectively. *Significant change from the baseline value, $p<0.0001$ (linear mixed-effects model). (a, b) Pairwise comparisons made by linear mixed-effects model adjusted for baseline values, pubertal stage, and sex, $p=0.0006,0.0286$, and 0.0064 for comparisons between Ca6D group and control, Ca3D, and Ca9D groups, respectively. b Change in serum IGF-1 from baseline (error bars

changes could be attributed to several aspects. First, the seasonal variations might contribute to fluctuations in serum $25(\mathrm{OH}) \mathrm{D}$ levels over time in our study as the baseline serum 25(OH)D levels were measured in fall (December 2010, Beijing, China) and measured again after one and a half years in early spring (May 2012, Beijing, China) [8]. Second, the relatively lower dose of vitamin D used in our study milk powder (approximately $300 \mathrm{IU} /$ day) was unlikely to improve the $25(\mathrm{OH}) \mathrm{D}$ level effectively. A randomized double-blind, placebo-controlled trial indicated that the $25(\mathrm{OH}) \mathrm{D}$ status significantly increased from 32 to $60 \mathrm{nmol} / \mathrm{L}$ and 28 to $46 \mathrm{nmol} / \mathrm{L}$ as results of 800 and $1600 \mathrm{IU} /$ day vitamin $\mathrm{D}_{3}$ supplementation for 5 months in schoolchildren with similar baseline vitamin D levels to our study; however, it is still not enough to correct vitamin D deficiency [15]. However, in another similar study, girls aged 10 years who received milk fortified with $133 \mathrm{IU}$ vitamin D and $245 \mathrm{mg}$ calcium per day had a significantly higher increase in the $25(\mathrm{OH}) \mathrm{D}$ level in comparison with those receiving milk fortified with only calcium $245 \mathrm{mg} / \mathrm{day}$ or no supplements [9]. The discrepancy

\section{$\square$ Control group $\mathbb{\mathbb { N } \text { Ca3D group }}$ \\ 血Ca6D group $\quad$ Ca9D group}

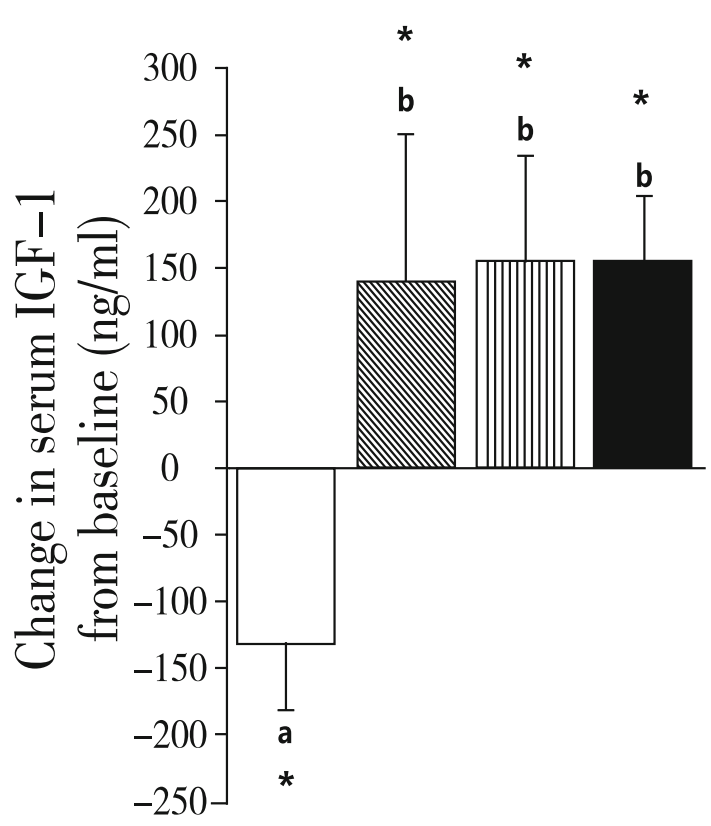

denote standard error). There was a significant group by time interaction and time effect, $p=0.0029$ and 0.0180 , respectively. $N=62,42,53$, and 47 for control, Ca3D, Ca6D, and Ca9D groups, respectively. *Significant change from the baseline value, $p<0.05$ (linear mixed-effects model). (a, b) Pairwise comparisons made by linear mixed-effects model adjusted for baseline values, pubertal stage, and sex, $p=0.0046,0.0029$, and 0.0022 for comparisons between the control group and Ca3D, Ca6D, Ca9D groups, respectively

might be due to different baseline $25(\mathrm{OH}) \mathrm{D}$ status, which in our study was almost one and a half times higher than that in that study [9].

\section{Effects on PTH, bone turnover, and bone density}

The role of calcium or milk supplementation in adolescents with insufficient baseline calcium intake is crucial in the improvement of bone mass acquisition [16-18]. As reported in a meta-analysis [4], there is only a trivial effect on bone mineral density, possibly due to the short supplementation duration of the majority of included studies with less than two years. Studies on the association of vitamin D intake or serum 25(OH)D status with bone density or bone mass had no consistent findings [19-25]. For instance, a previous study found that Chinese adolescent girls with insufficient vitamin D levels had significantly lower bone mass compared with those with sufficient vitamin D status [22], whereas this relationship was not found in some other studies [23-25]. A meta-analysis including six randomized controlled trials has suggested vitamin 
Table 4 Mixed linear model for changes of BMD and BMC at total body, lumbar spine, and total hip

Control $(N=73) \quad \operatorname{Ca3D}(N=49) \quad \operatorname{Ca} 6 \mathrm{D}(N=56) \quad \mathrm{Ca} 9 \mathrm{D}(N=54) \quad p$ value

Time $\quad$ Group by time

\begin{tabular}{|c|c|c|c|c|c|c|}
\hline \multicolumn{7}{|l|}{ Total body BMC (g) } \\
\hline Baseline & $1831.2 \pm 278.3$ & $1874.8 \pm 347.7$ & $1784.6 \pm 322.8$ & $1801.2 \pm 356.0$ & \multirow[t]{3}{*}{$<0.0001$} & \multirow[t]{3}{*}{0.5142} \\
\hline one year & $2039.9 \pm 281.9$ & $2071.4 \pm 309.4$ & $2015.3 \pm 334.3$ & $2004.5 \pm 399.2$ & & \\
\hline one and a half years & $2171.3 \pm 328.9$ & $2180.9 \pm 324.2$ & $2136.0 \pm 337.8$ & $2146.3 \pm 435.4$ & & \\
\hline \multicolumn{7}{|c|}{ Total body aBMD $\left(\mathrm{g} / \mathrm{cm}^{2}\right)$} \\
\hline Baseline & $0.749 \pm 0.071$ & $0.762 \pm 0.092$ & $0.733 \pm 0.076$ & $0.748 \pm 0.085$ & \multirow[t]{3}{*}{$<0.0001$} & \multirow[t]{3}{*}{0.4225} \\
\hline one year & $0.781 \pm 0.073$ & $0.787 \pm 0.090$ & $0.774 \pm 0.082$ & $0.779 \pm 0.097$ & & \\
\hline one and a half years & $0.816 \pm 0.089$ & $0.812 \pm 0.089$ & $0.810 \pm 0.094$ & $0.813 \pm 0.112$ & & \\
\hline \multicolumn{7}{|c|}{ Lumbar spine aBMD $\left(\mathrm{g} / \mathrm{cm}^{2}\right)$} \\
\hline Baseline & $0.812 \pm 0.139$ & $0.816 \pm 0.170$ & $0.781 \pm 0.131$ & $0.805 \pm 0.127$ & \multirow[t]{3}{*}{$<0.0001$} & \multirow[t]{3}{*}{0.6132} \\
\hline one year & $0.918 \pm 0.169$ & $0.918 \pm 0.165$ & $0.869 \pm 0.160$ & $0.895 \pm 0.169$ & & \\
\hline one and a half years & $0.952 \pm 0.135$ & $0.946 \pm 0.164$ & $0.933 \pm 0.153$ & $0.937 \pm 0.138$ & & \\
\hline \multicolumn{7}{|l|}{ Total hip aBMD $\left(\mathrm{g} / \mathrm{cm}^{2}\right)$} \\
\hline Baseline & $0.845 \pm 0.102$ & $0.855 \pm 0.131$ & $0.837 \pm 0.118$ & $0.856 \pm 0.108$ & \multirow[t]{3}{*}{$<0.0001$} & \multirow[t]{3}{*}{0.1780} \\
\hline one year & $0.870 \pm 0.108$ & $0.889 \pm 0.124$ & $0.856 \pm 0.116$ & $0.865 \pm 0.120$ & & \\
\hline one and a half years & $0.892 \pm 0.124$ & $0.888 \pm 0.123$ & $0.900 \pm 0.150$ & $0.892 \pm 0.133$ & & \\
\hline
\end{tabular}

$N=54,45,48$, and 51 for control, Ca3D, Ca6D, and Ca9D groups at one year

$N=58,36,45$, and 42 for control, Ca3D, Ca6D, and Ca9D groups at one and a half years

There were no significant differences in baseline values between groups

Adjusted for baseline value, sex, and baseline pubertal stage

There were significant differences from baseline at one and one and a half years for all values in each group, $p<0.05$

D supplements are not likely to benefit BMD or BMC in children and adolescents with normal vitamin D levels but in those with deficient vitamin D levels [10]. The findings of the metaanalysis remain unconfirmed possibly due to several reasons, including the less number of included studies, the lower doses of vitamin D supplements, the statistical power (two of six studies had only 26 and 98 participants), and the short supplementation duration (four of six studies are only one year).

Studies relevant to the interaction of vitamin D and calcium are limited. In our study, we did not find the treatment effect on $\mathrm{BMC}$ or $\mathrm{BMD}$ in any site during the one and a half-year intervention period. A possible explanation is that non-significantly changed serum 25(OH)D levels resulted from insufficient vitamin D supplements and low baseline vitamin D status, despite sufficient calcium supplementation. One previous study found that low calcium diet and low $25(\mathrm{OH}) \mathrm{D}$ status had a negative interaction on the lumbar spine BMC and BMD [8], which might imply that a low serum $25(\mathrm{OH}) \mathrm{D}$ status would aggravate the adverse impact of deficient calcium. In the present study, we did not find significant intervention effects on ALP and TRAP$5 \mathrm{~b}$, whereas significantly increased serum ALP concentration was observed only in the Ca6D group while TRAP-5b levels increased significantly over time in all groups. This might be explained by different pubertal stages between groups as, at baseline, almost $50 \%$ of participants in the Ca6D group were at stage one and two, at which, however, only about $35 \%$ of participants in both Ca3D and Ca9D groups were [8]. In addition, the serum PTH level was significantly decreased by intervention only in the Ca6D group, although it was also decreased in two other intervention groups. The highest baseline PTH level and the lowest 25(OH)D level and the intervention could be the main reasons. In contrast, Esterle et al. indicated that the increased bone turnover was not evidenced to be the cause of lower lumbar spine mineralization in late-pubertal girls with low calcium and $25(\mathrm{OH}) \mathrm{D}$. This study further suggested that increased PTH level was not likely to explain the lower lumbar spine aBMD as which was only found in participants with a high PTH level and low calcium and 25(OH)D status, whereas not in those with high calcium status. We, thus, proposed that comprehensive evaluation on calcium, vitamin $\mathrm{D}$, and $\mathrm{PTH}$ levels might be necessary to determine their optimal level to bone health.

\section{Effects on IGF-1}

Our study showed a significant intervention effect on IGF-1, which increased in all intervention groups while decreased in the control group. This finding was consistent with a crosssectional study suggesting a significantly positive association between energy-adjusted calcium intake and IGF-1 in 
adolescent females (adjusted $r=0.24, p<0.05$ ) [26]. In addition, it was evident that average serum IGF-1 levels in the present study at baseline (667 to $789 \mathrm{ng} / \mathrm{mL}$ for all groups) were higher than those in other studies (median ranged from 275 to $450 \mathrm{ng} / \mathrm{mL}$ ) in terms of adolescents aged 12 to 15 years [27-29]. This difference might explain that no dose-response relationship between calcium supplement and change in the IGF-1 level was observed in our study in spite of low baseline calcium intake and different calcium supplement. Studies in both adults [30] and adolescents [26] found that the IGF-1 level was also associated with protein intake, which was also supplemented in the milk powder used in our study. The positive association between protein intake and IGF-1 level was therefore supported by our results to some degree.

\section{Strengths and limitations}

Several strengths of our study are randomized controlled design, high retention and compliance rates, and recruited participants who can represent healthy pubertal school-aged adolescents in China. A limitation of the present study is the limited funding and other markers of bone formation have not been detected, such as those recommended by IOF/ IFCC: bone formation marker (s-PINP) and bone resorption marker (s-CTX) [31]. Another limitation is that participants had relatively high IGF-1 levels at baseline which could have an impact on the effect of increased IGF-1 in study groups on bone health. The third limitation is that relatively low vitamin D supplements could limit the effect of calcium supplements on bone mineralization and bone mineral accrual. Therefore, higher vitamin $\mathrm{D}$ and calcium intervention could be the focus of future research in healthy adolescents with a moderate IGF1 level and low vitamin D and calcium status.

\section{Conclusions}

In conclusion, in these healthy pubertal adolescents with low vitamin $\mathrm{D}$ and calcium status, calcium and vitamin D-fortified milk powder supplementation did not have a significant effect on bone turnover and mineralization. IGF-1 levels were improved by intervention in spite of high baseline status.

Acknowledgments The most important acknowledgment is to the participants in the study and the hard work of every staff member, as well as the strong support of the school leaders.

Funding This work was financially supported by the Chinese Statesupporting Technology Project (2008BAI58B02).

\section{Compliance with ethical standards}

The trial was approved by the ethics committee of the Institute for Nutrition and Food Safety, Chinese Center for Disease Control and Prevention (now renamed the Ethics Committee of the National
Institute of Nutrition and Health, Chinese Center for Disease Control and Prevention). The investigators explained the benefits and risks of participation in the trial to each participant and their parents. A written informed consent and assent form was signed by each participant's parents prior to the initiation of the survey.

Conflicts of interest The authors declare that they have no conflict of interest.

Open Access This article is distributed under the terms of the Creative Commons Attribution-NonCommercial 4.0 International License (http:// creativecommons.org/licenses/by-nc/4.0/), which permits any noncommercial use, distribution, and reproduction in any medium, provided you give appropriate credit to the original author(s) and the source, provide a link to the Creative Commons license, and indicate if changes were made.

\section{References}

1. Huncharek M, Muscat J, Kupelnick B (2008) Impact of dairy products and dietary calcium on bone-mineral content in children: results of a meta-analysis. Bone 43(2):312-321

2. Golden NH, Abrams SA (2014) Optimizing bone health in children and adolescents. Pediatrics 134(4):e1229-e1243

3. Cameron MA, Paton LM, Nowson CA, Margerison C, Frame M, Wark JD (2004) The effect of calcium supplementation on bone density in premenarcheal females: a co-twin approach. J Clin Endocrinol Metab 89(10):4916-4922

4. Winzenberg T, Shaw K, Fryer J, Jones G (2006) Effects of calcium supplementation on bone density in healthy children: meta-analysis of randomised controlled trials. Bmj 333(7572):775

5. Lambert HL, Eastell R, Karnik K, Russell JM, Barker ME (2008) Calcium supplementation and bone mineral accretion in adolescent girls: an 18-mo randomized controlled trial with 2-y follow-up. Am J Clin Nutr 87(2):455-462

6. Davies JH, Evans BA, Gregory JW (2005) Bone mass acquisition in healthy children. Arch Dis Child 90(4):373-378

7. Lehtonen-Veromaa MK, Mottonen TT, Nuotio IO, Irjala KM, Leino AE, Viikari JS (2002) Vitamin D and attainment of peak bone mass among peripubertal Finnish girls: a 3-y prospective study. Am J Clin Nutr 76(6):1446-1453

8. Esterle L, Nguyen M, Walrant-Debray O, Sabatier JP, Garabedian M (2010) Adverse interaction of low-calcium diet and low $25(\mathrm{OH}) \mathrm{D}$ levels on lumbar spine mineralization in late-pubertal girls. J Bone Miner Res 25(11):2392-2398

9. Du X, Zhu K, Trube A, Zhang Q, Ma G, Hu X, Fraser DR, Greenfield H (2004) School-milk intervention trial enhances growth and bone mineral accretion in Chinese girls aged 10-12 years in Beijing. Br J Nutr 92(1):159-168

10. Winzenberg T, Powell S, Shaw KA, Jones G (2011) Effects of vitamin $\mathrm{D}$ supplementation on bone density in healthy children: systematic review and meta-analysis. Bmj 342:c7254

11. Yang Y, Wang G, Pang X (2002) China food composition 2002. Peking University Medical Press, Beijing, p 2002

12. Yang Y (2004) China food composition 2004-Book2. Peking University Medical Press, Beijing, p 2004

13. Society CN (2000) Chinese DRIs. Chinese Light Industry Publishing House, Beijing

14. Holick MF (2007) Vitamin D deficiency. N Engl J Med 357(3): 266-281

15. Ghazi AA, Hosseinpanah F, E MA, Ghazi S, Hedayati M, Azizi F (2010) Effects of different doses of oral cholecalciferol on serum 
25(OH)D, PTH, calcium and bone markers during fall and winter in schoolchildren. Eur J Clin Nutr 64(12):1415-1422

16. Prentice A, Ginty F, Stear SJ, Jones SC, Laskey MA, Cole TJ (2005) Calcium supplementation increases stature and bone mineral mass of 16- to 18-year-old boys. J Clin Endocrinol Metab 90(6): 3153-3161

17. Johnston CC Jr, Miller JZ, Slemenda CW, Reister TK, Hui S, Christian JC, Peacock M (1992) Calcium supplementation and increases in bone mineral density in children. N Engl J Med 327(2): 82-87

18. Cadogan J, Eastell R, Jones N, Barker ME (1997) Milk intake and bone mineral acquisition in adolescent girls: randomised, controlled intervention trial. Bmj 315(7118):1255-1260

19. Outila TA, Karkkainen MU, Lamberg-Allardt CJ (2001) Vitamin D status affects serum parathyroid hormone concentrations during winter in female adolescents: associations with forearm bone mineral density. Am J Clin Nutr 74(2):206-210

20. Cheung TF, Cheuk KY, Yu FW, Hung VW, Ho CS, Zhu TY, Ng BK, Lee KM, Qin L, Ho SS, Wong GW, Cheng JC, Lam TP (2016) Prevalence of vitamin D insufficiency among adolescents and its correlation with bone parameters using high-resolution peripheral quantitative computed tomography. Osteoporos Int 27(8):24772488

21. Cashman KD, Hill TR, Cotter AA, Boreham CA, Dubitzky W, Murray L, Strain J, Flynn A, Robson PJ, Wallace JM, Kiely M (2008) Low vitamin D status adversely affects bone health parameters in adolescents. Am J Clin Nutr 87(4):1039-1044

22. Foo LH, Zhang Q, Zhu K, Ma G, Hu X, Greenfield H, Fraser DR (2009) Low vitamin D status has an adverse influence on bone mass, bone turnover, and muscle strength in Chinese adolescent girls. J Nutr 139(5):1002-1007

23. Kristinsson JO, Valdimarsson O, Sigurdsson G, Franzson L, Olafsson I, Steingrimsdottir L (1998) Serum 25-hydroxyvitamin $\mathrm{D}$ levels and bone mineral density in 16-20 years-old girls: lack of association. J Intern Med 243(5):381-388

24. Oliveri MB, Wittich A, Mautalen C, Chaperon A, Kizlansky A (2000) Peripheral bone mass is not affected by winter vitamin D deficiency in children and young adults from Ushuaia. Calcif Tissue Int 67(3):220-224
25. Willett AM (2005) Vitamin D status and its relationship with parathyroid hormone and bone mineral status in older adolescents. Proc Nutr Soc 64(2):193-203

26. Kerver JM, Gardiner JC, Dorgan JF, Rosen CJ, Velie EM (2010) Dietary predictors of the insulin-like growth factor system in adolescent females: results from the Dietary Intervention Study in Children (DISC). Am J Clin Nutr 91(3):643-650

27. Isojima T, Shimatsu A, Yokoya S, Chihara K, Tanaka T, Hizuka N, Teramoto A, Tatsumi KI, Tachibana K, Katsumata N, Horikawa R (2012) Standardized centile curves and reference intervals of serum insulin-like growth factor-I (IGF-I) levels in a normal Japanese population using the LMS method. Endocr J 59(9):771-780

28. Hyun SE, Lee BC, Suh BK, Chung SC, Ko CW, Kim HS, Lee KH, Yang SW, Shin CH, Hwang JS, Kim DH, Lim BK, Kim JD, Yoo HW, Kim HS, Chung WY, Park MJ, Woo YJ, Kim CJ, Lee DY, Kim EY, Choi JH, Han HS, Hwang IT, Kim HS (2012) Reference values for serum levels of insulin-like growth factor-I and insulinlike growth factor binding protein-3 in Korean children and adolescents. Clin Biochem 45(1-2):16-21

29. Bedogni G, Giannone G, Maghnie M, Giacomozzi C, Di Iorgi N, Pedicelli S, Peschiaroli E, Melioli G, Muraca M, Cappa M, Cianfarani S (2012) Serum insulin-like growth factor-I (IGF-I) reference ranges for chemiluminescence assay in childhood and adolescence. Data from a population of in- and out-patients. Growth Hormon IGF Res 22(3-4):134-138

30. Zhu K, Meng X, Kerr DA, Devine A, Solah V, Binns CW, Prince RL (2011) The effects of a two-year randomized, controlled trial of whey protein supplementation on bone structure, IGF-1, and urinary calcium excretion in older postmenopausal women. J Bone Miner Res 26(9):2298-2306

31. Vasikaran S, Eastell R, Bruyère O, Foldes AJ, Garnero P, Griesmacher A, McClung M, Morris HA, Silverman S, Trenti T, Wahl DA, Cooper C, Kanis JA, IOF-IFCC Bone Marker Standards Working Group (2011) Markers of bone turnover for the prediction of fracture risk and monitoring of osteoporosis treatment: a need for international reference standards. Osteoporos Int 22(2):391-420

Publisher's note Springer Nature remains neutral with regard to jurisdictional claims in published maps and institutional affiliations. 